\section{Sensory Ataxia}

Kerry Donnelly

VA WNY Healthcare System, University of Buffalo (SUNY) Behavioral Health Careline (116B), Buffalo, NY, USA

\section{Definition}

Sensory ataxia is a problem of incoordination resulting from a disruption of somatosensory feedback.

\section{Current Knowledge}

Sensory ataxia is both a sign and a symptom in neurology. Although one may not always be consciously aware of it, motor activities - especially those involving the coordination of either upper or lower extremities - rely heavily on sensory input, especially kinesthetic and proprioceptive feedback. While visual feedback (looking at the limbs) during movements can be of some assistance, vision alone is inadequate in many situations. Thus, sensory ataxia is the result of disruption of neuronal pathways that interfere with proprioception, particularly lesions involving peripheral nerves, dorsal nerve roots, posterior (dorsal) columns, or the medial lemniscus. Cortical (parietal) lesions may produce similar deficits, but less commonly. Sensory ataxia is distinguished from other types of ataxia by marked worsening of coordination when the eyes are closed. Patients with this condition often walk with a broad-based gait and stand with their legs apart. They may complain that they find it difficult or near impossible to walk in a darkened room or losing their balance when closing their eyes in the shower or removing clothes over their head. A routine test for this condition is to ask the patient to stand with their feet together and then continue to do so after closing their eyes. Marked difficulty in doing so (a "positive Romberg") is suggestive of loss of proprioceptive feedback. Asking a patient to flex their arms at their elbows and rapidly rotate their closed fists around each other (with their eyes closed) may demonstrate upper limb ataxia (fists may keep bumping into one another).

\section{Cross-References}

\section{Ataxia \\ - Proprioception}

\section{References and Readings}

Mendoza, J.E., \& Foundas, A.L. (2008). The somatosensory systems. In Clinical neuroanatomy: A behavioral approach (pp. 23-47). New York: Springer.

Sobue, G. (1998). Acute and chronic sensory neuronopathy. In N. Latov, J. H. Wokke, \& J. J. Kelly (Eds.), Immunological and infectious diseases of the peripheral nerves (p. 129). New York: Cambridge University Press. 